\title{
Drug Accountability Domain
}

National Cancer Institute

\section{Source}

National Cancer Institute. Drug Accountability Domain. NCI Thesaurus. Code C49578.

A subject domain utilized for the submission of information encompassing and

representing data, vocabulary or records related to drug accountability. 